Revista Calidad en la Educación Superior

Programa de Autoevaluación Académica

Universidad Estatal a Distancia, Costa Rica

ISSN 1659-4703

revistacalidad@uned.ac.cr

\title{
ANÁLISIS DE LA FORMACIÓN PROFESIONAL DE LOS DIRECTORES GRADUADOS Y LAS DIRECTORAS GRADUADAS DE LA CARRERA DE ADMINISTRACIÓN EDUCATIVA DEL CIDE-UNA
}

\author{
ANALYSIS FORMAL TRAINING FROM THE PRINCIPALS GRADUATES \\ FROM EDUCATIONAL ADMINISTRATION AT CIDE FROM UNIVERSIDAD \\ NACIONAL
}

\author{
lleana Vargas Jiménez ${ }^{1}$ \\ ileana.vargas.jimenez@una.cr \\ Investigadora del CIDE \\ Universidad Nacional, Costa Rica
}

\author{
Volumen 6, número 1 \\ Mayo 2015 \\ Pp. 174 - 212
}

Recibido: 28 de agosto, 2014

Aprobado: 27 de febrero, 2015

\footnotetext{
${ }^{1}$ lleana Vargas Jiménez, Doctora, investigadora y profesora de la División de Educación para el Trabajo, Centro de Investigación y Docencia en Educación (CIDE).
} 
Análisis de la formación profesional de los directores graduados y las directoras graduadas de la carrera de Administración Educativa del CIDE-UNA

lleana Vargas Jiménez

\section{Resumen}

La investigación tiene como objetivo valorar la coherencia entre la formación profesional recibida por las personas graduadas en Administración Educativa del CIDE y el desempeño laboral en los contextos educativos, teniendo en cuenta los procesos de comunicación, la toma de decisiones y el ejercicio del liderazgo desde el plan de estudios de la carrera. Este trabajo es un estudio descriptivo que utiliza el enfoque cuantitativo. La recolección de los datos se llevó a cabo mediante un cuestionario y el análisis de los mismos se realiza por medio del SPSS. (Paquete estadístico para Ciencias Sociales).

Las personas participantes son las graduadas de la carrera de Administración Educativa del CIDE, Universidad Nacional, en el periodo 2000-2011 y quienes hayan ejercido o estén en un puesto de dirección, asistencia administrativa o subdirección.

Entre las principales conclusiones se destacan la relación existente entre la formación profesional y la práctica directiva y la incidencia de los aprendizajes logrados a lo largo de la carrera en el desempeño administrativo.

Palabras claves: formación profesional, práctica directiva, liderazgo, procesos de comunicación, toma de decisiones, administración educativa

\section{Abstract}

The study aimed to assess the consistency between the training received by the CIDE's educational administration graduates and the job performance in educational contexts, taking into account the processes of communication, decision-making and the leadership exercise that take place at the institutions fromthe studywas used with the quantitative approach with a descriptive method. The data collection was made through questionnaires and it was proceeded with the use of the SPSS program.

The participants who attended were the graduates from Educational Administration at CIDE from the Universidad Nacional, from 2000 to 2011 and who have exercised or holds a position as principal, assistant principal or administrative assistant. According to the research results there is a relationship between the formal training of an education administrator. According to the graduates formal training is fundamental for their professional performance.

Keywords: formal training, practice, leadership, communication processes, decision-makingskills, good management performance, education administration

El área temática de esta investigación está vinculada a factores del desempeño profesional de los directores escolares graduados de la Universidad Nacional de Heredia. Por lo tanto, me interesa indagar sobre el nivel de satisfacción que 
Análisis de la formación profesional de los directores graduados y las directoras graduadas de la carrera de Administración Educativa del CIDE-UNA

sienten las personas graduadas en el periodo 2000-2011 en cuanto a su proceso de aprendizaje en la carrera.

Según el informe Estado de la Educación 2 (2008), desde la década de 1950 ha sido motivo de preocupación para los altos niveles de conducción del sistema educativo costarricense la formación y profesionalización de las personas responsables de los roles administrativos-docentes, por ser agentes privilegiados de cambio en la realidad educativa.

Las instituciones escolares y las personas responsables de dirigirlas no están exentas del cambio vertiginoso que se está dando en la actualidad. En ese sentido, la persona que dirige es la autoridad formal de la institución educativa, se espera que administre de forma competente y que cuente con una adecuada preparación universitaria que le proporcione no solo las herramientas necesarias para cumplir apropiadamente con su trabajo, sino también la preparación académica indicada.

En el ya mencionado Estado de la Educación 2 (2008) se señala que las funciones del director o de la directora de un centro educativo, según el Manual de Puestos del Ministerio de Educación Pública, son las siguientes:

I. Planea, dirige, coordina y supervisa las actividades curriculares y administrativas de la institución a su cargo.

II. Asesora y orienta al personal en aspectos curriculares y administrativos, procurando la incorporación de conocimientos actualizados e innovadores.

III. Coordina los diferentes programas del centro educativo y vela por su correcta ejecución.

IV. Organiza y dirige actividades administrativas y culturales cívicas y sociales. 
Análisis de la formación profesional de los directores graduados y las directoras graduadas de la carrera de Administración Educativa del CIDE-UNA

lleana Vargas Jiménez

De acuerdo a lo anterior, se puede constatar que debe asumir muchas tareas probablemente con escaso tiempo para llevarlas a cabo de manera adecuada (Programa Estado de la Nación en Desarrollo Humano Sostenible, 2008).

Asimismo, el Ministerio de Educación Pública, en el documento Diez Líneas Estratégicas 2010-2014 (2010), defiende y señala claramente en una de sus líneas estratégicas «el centro educativo de calidad eje de la educación costarricense", lineamiento en el que pretende colocar al centro escolar como punto de partida, dándoles un papel protagónico al director y a la comunidad educativa.

El currículo nacional básico debe adaptarse a las poblaciones de nuestro país y lograr que la comunidad estudiantil aprenda lo que es relevante y que lo aprenda bien. Si lo logramos habremos cumplido con el objetivo principal de toda persona educadora: que nuestra población infantil y juvenil aprenda las destrezas básicas necesarias y que aprenda a vivir y convivir, es decir, es un reto que garantizaría una educación pública de calidad.

Además, es esencial promover el empoderamiento de los directores contribuyendo a fomentar la identidad como la calidad de cada centro educativo, sin olvidar la propia cultura de las escuelas, los principios, los valores y las creencias compartidas que le dan una identidad propia y que explican los comportamientos de los individuos en la misma institución, siendo un elemento fundamental para el desarrollo central de una gestión organizacional eficaz y adecuada.

Es un desafío integrar a docentes y directores en espacios de interacción que les permitan reflexionar más allá de las necesidades disciplinarias, estos espacios deben surgir de reglamentaciones que emanen del Ministerio de Educación Pública. 
Análisis de la formación profesional de los directores graduados y las directoras graduadas de la carrera de Administración Educativa del CIDE-UNA

lleana Vargas Jiménez

Lo que se requiere es hacer de las instituciones escolares comunidades educativas en las cuales lo vital sean los procesos de enseñanza y aprendizaje de la población estudiantil (Organización de Estados Iberoamericanos para la Educación, la Ciencia y la Cultura, 2010).

De mantenerse la situación actual en las instituciones educativas los pronósticos serán desalentadores, debido al incremento de la inequidad y a la ausencia de calidad en los procesos de enseñanza-aprendizaje.

Tal como lo señaló López (2005), la diversidad de escenarios, las múltiples expresiones de pobreza y las nuevas formas de exclusión social y espacial dan como resultado sociedades más fragmentadas y una coexistencia de múltiples configuraciones culturales (especialmente juveniles); estos son los elementos constitutivos del nuevo panorama social de América Latina y, por ende, de Costa Rica.

Según López (2005), la frustración no solo la viven los padres y las madres con respecto a la educación que reciben sus hijos e hijas, sino también la población docente, debido a las condiciones en las que trabaja y los resultados que logra en sus estudiantes. De tal manera, con el trabajo planteado se pretende identificar la formación profesional del director y de la directora escolares desde la toma de decisiones, los procesos de comunicación y el ejercicio del liderazgo.

Se espera lograr un conocimiento adicional al que se ha desarrollado en la División de Educación para el Trabajo, cuyo interés radica no solo en el fortalecimiento del plan de estudios de Administración Educativa, sino también en los tomadores de decisiones del Ministerio de Educación Pública en torno al trabajo que llevan a cabo los directores escolares. 
Análisis de la formación profesional de los directores graduados y las directoras graduadas de la carrera de Administración Educativa del CIDE-UNA

\section{Planteamiento del problema}

Me interesa indagar sobre la formación profesional de las personas egresadas de la carrera de Administración Educativa del Centro de Investigación y Docencia en Educación, adscrita a la Universidad Nacional. Es fundamental determinar el nivel de satisfacción que la población seleccionada siente respecto a su carrera y al plan de estudios, para ello se quiere trabajar desde tres ejes esenciales para todo administrador educativo: la toma de decisiones, el proceso de comunicación que acompaña siempre al director o a la directora escolar y el ejercicio del liderazgo.

Sin embargo, cabe mencionar que existen problemas dentro de las tareas administrativas, tal como lo evidencia el informe Estado de la Educación 2 (2008):

I. Excesiva centralización del sistema educativo

II. Gran cantidad de trámites que deben realizarse

III. Pocos recursos humanos y financieros con los que cuentan algunos centros educativos.

Por lo anterior, las personas integrantes de los centros escolares experimentan frustración, bajos niveles de logro y desmotivación hacia el logro educativo, esto genera poca productividad en las metas planteadas, siendo concordante con los resultados de investigaciones que se señalaron en el primer apartado de este trabajo. En las investigaciones abordadas se señala que la escuela y particularmente la persona gestora, como también se le llama al director o a la directora, es la responsable directa del trabajo que se lleva a cabo en el plantel, de él o ella depende el trabajo que se lleva a cabo y los resultados.

Se ha reiterado que hoy más que nunca la escuela debe responder con procesos inclusivos y con programas de calidad; de no prestar atención a la problemática se continuará con resultados lamentables, especialmente para las escuelas públicas de Costa Rica y para la formación de administradores educativos desde la universidad. Siendo una relación causal, según Cano (2009) y Vargas (2010). 
Análisis de la formación profesional de los directores graduados y las directoras graduadas de la carrera de Administración Educativa del CIDE-UNA

lleana Vargas Jiménez

Asimismo, las tendencias mundiales señalan que la preparación profesional del gestor educativo es esencial, aunado al papel que debe representar para el logro de los objetivos institucionales.

La escuela es la institución por excelencia en la que debe centrarse el cambio y la mejora institucional. Esto coincide con las investigaciones de Barrera, Fasih y Patrinos (2009) y Concha (2007).

De tal manera, contar con una identificación de la formación docente desde los directores y las directoras escolares que egresaron de esta universidad pública resulta de especial importancia para el CIDE. Concretamente para la División de Educación para el Trabajo, ya que no se han realizado investigaciones de esta naturaleza en el ámbito universitario. Además de exponer los resultados en esta investigación, estos podrán ser tomados en cuenta para enriquecer no solo el plan de estudios de la Licenciatura en Administración Educativa de la unidad académica aludida, sino para la toma de decisiones de las personas encargadas del Ministerio de Educación Pública, por cuanto el perfil del administrador educativo debe responder a lo que demanda la sociedad nacional.

De no llevarse a cabo esta indagación se estaría perdiendo una valiosa oportunidad académica para enriquecer la oferta académica de la Licenciatura en Administración Educativa. Es importante contar con información única tendiente a la formación docente de las personas egresadas de la carrera de Administraron Educativa, información válida y confiable para la toma de decisiones en torno a los planes de estudio y para los tomadores de decisiones del Ministerio de Educación, con el fin de contar con un panorama útil y confiable acerca de las funciones que este grupo de profesionales llevan a cabo y cómo las enfrentan. Ante este panorama se presenta la formulación del problema de investigación. 
Análisis de la formación profesional de los directores graduados y las directoras graduadas de la carrera de Administración Educativa del CIDE-UNA

\begin{tabular}{|l|l|}
\hline \multicolumn{1}{|c|}{ Problema de investigación } & \multicolumn{2}{c|}{ Pregunta de investigación } \\
\hline ¿Cuál es la relación entre la formación & ¿Cómo incide la formación \\
profesional recibida y la práctica directiva & profesional recibida por los \\
escolar de la persona graduada de & directores y las directoras que se \\
Administración Educativa del CIDE, UNA, & graduaron en Administración \\
desde los procesos de toma de & Educativa del CIDE en su labor \\
decisiones, el ejercicio del liderazgo y los & administrativa en las instituciones \\
procesos de comunicación? & educativas? \\
\hline
\end{tabular}

\section{Hipótesis de la investigación}

A continuación se expone la hipótesis que dirige el presente estudio:

El plan de estudios de la carrera de Administración Educativa del CIDE- UNA no proporciona los requerimientos necesarios para que el director o la directora desempeñe una adecuada práctica directiva desde la mirada de los actores involucrados del proceso.

\section{Los objetivos de la investigación}

Se plantean a continuación los objetivos de la investigación en correspondencia con lo planteado anteriormente.

\begin{tabular}{|c|c|}
\hline Objetivo general & Objetivo específico \\
\hline $\begin{array}{l}\text { Valorar la coherencia entre la formación } \\
\text { profesional recibida por las personas } \\
\text { graduadas en Administración Educativa } \\
\text { del CIDE y el desempeño laboral en los } \\
\text { contextos educativos, teniendo en cuenta } \\
\text { los procesos de toma de decisiones, el } \\
\text { ejercicio del liderazgo y los procesos de } \\
\text { comunicación que se dan en las } \\
\text { instituciones del estudio. }\end{array}$ & $\begin{array}{l}\text { Analizar la coherencia del plan de } \\
\text { estudios de Administración } \\
\text { Educativa del CIDE-UNA con base } \\
\text { en el criterio de personas } \\
\text { graduadas de la carrera }\end{array}$ \\
\hline
\end{tabular}

\section{Antecedentes teóricos}

En los estudios consultados para la elaboración de este trabajo se encontraron coincidencias en torno a la propuesta de investigar sobre la formación profesional, especialmente en el campo de la administración educativa, siendo hoy uno de los principales retos que se deben enfrentar desde las 
Análisis de la formación profesional de los directores graduados y las directoras graduadas de la carrera de Administración Educativa del CIDE-UNA

lleana Vargas Jiménez

universidades, que tienen bajo su responsabilidad la formación de administradores educativos, con el fin de mejorar la calidad, la inclusión y la equidad de la educación.

Es así que se comparten resultados de investigaciones llevadas a cabo en Europa, Estados Unidos y América Latina en cuanto a los procesos de formación profesional docente, la efectividad docente y escolar, la administración educativa y de gestión y el ejercicio profesional.

Se encontraron resultados en relación con los bajos perfiles académicos. Para solucionar esta problemática se debe procurar movilizar el conocimiento teórico a situaciones concretas y rescatar los procesos de integración entre la salida de la formación inicial y la entrada al trabajo docente. Asimismo, los análisis de los modelos de gestión de la formación docente universitaria demostraron que es cada vez mayor el rol que juegan el director y sus equipos directivos en la formación de directores y en la gestión del centro escolar.

Se debe investigar aún más sobre la efectividad escolar con el propósito fundamental de establecer políticas públicas que garanticen equidad y calidad educativas, según Cuenca (2005) y Pogré (2004).

Cabe destacar que en estas investigaciones se emplea la entrevista estructurada, el estudio de caso, la entrevista etnográfica en profundidad, la observación directa de las prácticas, análisis de documentos, encuestas, grupos focales, entre otros. Los enfoques utilizados son el cualitativo, el cuantitativo, la investigación aplicada de tipo descriptivo, el expo facto, y el descriptivo-interpretativo.

La formación permanente es eventual y es asumida por los ministerios de educación de los países de América Latina, lo que no permite que las demandas del contexto sean atendidas. Se requiere una formación permanente 
Análisis de la formación profesional de los directores graduados y las directoras graduadas de la carrera de Administración Educativa del CIDE-UNA

lleana Vargas Jiménez

especializada de la persona directora y la integración de docentes en equipos organizados (Vázquez y Angulo, 2006; Garbanzo y Orozco, 2010)

Concha (2007) manifestó que la formación del director es cada vez más evidente y resulta fundamental para alcanzar metas, mejorar las relaciones interpersonales director-docente y orientar el proceso de interacción hacia la mejora, la participación y la toma de decisiones.

Fortalecer los planes de estudio de formación profesional y el ambiente laboral es determinante para el desempeño laboral; se plantea la necesidad de diseñar un nuevo modelo, más participativo y comprometido a nivel profesional y de desarrollo (Cunningham y Sherman, 2008).

Por otra parte, de acuerdo con Tello (2008), es necesario lograr una mayor autonomía en el profesional por medio de sistemas de alto desempeño: advertirle sobre sus debilidades, suministrarle conocimientos precisos de mejores prácticas, motivarlo.

Villarreal (2005) manifestó que si no se cuenta con un director eficiente las escuelas tienen pocas probabilidades de poseer una cultura con altas expectativas. Por lo tanto, se requieren retos de cobertura, equidad y calidad y la demanda de programas innovadores y con iniciativas. Se ratifica que un fuerte liderazgo es relevante a la hora de lograr mejoras en las instituciones escolares.

La administración educativa debe cambiar de rumbo, consolidando nuevos modelos de organización y gestión de centros escolares (Angulo, Hidalgo, Martínez, Mora y Ramírez, 2009). Esto significa que es primordial lograr una comprensión profunda de la realidad que involucre al director escolar, así como a las personas que actúan, sienten y piensan en torno al desempeño y competencias profesionales de las personas directoras. 
Análisis de la formación profesional de los directores graduados y las directoras graduadas de la carrera de Administración Educativa del CIDE-UNA

lleana Vargas Jiménez

Aún falta por investigar en el contexto nacional sobre la formación profesional que reciben los administradores educativos en la universidad. En Costa Rica la formación docente de los administradores educativos le corresponde a tres universidades públicas: la Universidad Estatal a Distancia, la Universidad de Costa Rica y la Universidad Nacional.

En esta investigación me interesa indagar desde la Universidad Nacional, y específicamente desde el Centro de Investigación y Docencia en Educación (CIDE), el quehacer de los directores desde su cotidianidad y poder determinar si la formación académica responde a las demandas de su tiempo. Para ello es relevante investigar si la formación docente recibida responde a su práctica directiva desde tres variables: la toma de decisiones, el ejercicio del liderazgo y los procesos de comunicación.

Se logra evidenciar a lo largo de los antecedentes señalados que estas investigaciones se han concentrado en la formación docente y de alguna manera se han excluido los aspectos de formación profesional de los administradores educativos, especialmente a nivel nacional. Además, se sabe por los resultados aportados que las consecuencias demuestran que el campo de la administración educativa, el de la formación profesional en particular, es poco abordado; no se encontraron investigaciones que permitieran profundizar en el tema de la administración educativa y la formación docente a nivel nacional.

Constantemente se ha ido apostando a que para comprender el mundo escolar es necesario conocer y analizar las características, dinámicas, variables y factores presentes al interior de la escuela (Latorre, 2006).

\section{Marco metodológico}

\section{Descripción de la metodología y el diseño de investigación}

Para la fase cuantitativa de la investigación se utiliza un enfoque descriptivo, ya que se detallan fenómenos, situaciones, contextos y eventos, y cómo estos se 
Análisis de la formación profesional de los directores graduados y las directoras graduadas de la carrera de Administración Educativa del CIDE-UNA

lleana Vargas Jiménez

manifiestan, según lo planteado por Hernández, Fernández y Baptista (2006). Los estudios descriptivos buscan especificar las propiedades, las características y los rasgos importantes de cualquier fenómeno que se analice; en este caso, son las variables de formación profesional y la práctica directiva, cuya dimensión se mide en el ejercicio del liderazgo, la toma de decisiones y los procesos de comunicación que ejerce el director o la directora en su institución.

\section{Definición operativa y conceptual de las variables del estudio}

La construcción operativa del instrumento se fundamenta en la definición sustantiva de dos variables: la formación profesional y la práctica directiva, y se dimensionan en procesos de comunicación, toma de decisiones, ejercicio del liderazgo, habilidades para el desempeño profesional y valores.

\section{Formación profesional}

La formación profesional es aquella con carácter holístico en la que el o la estudiante debe adquirir autonomía intelectual, capacidad crítica de su entorno y capacidad para resolver problemas, para aprehender su papel social y aplicarlo en su práctica directiva (Vargas, Pacheco y Villalobos, 2004; Cuenca, 2012). La formación profesional implica el desarrollo de competencias necesarias para realizar la actividad profesional.

\section{Práctica directiva}

Es la conjunción de percepciones, creencias y formas de pensar con las que se lleva a cabo el trabajo diario en la institución escolar (Latorre, 2006), a través del liderazgo, de los procesos de comunicación y de la toma de decisiones (Vargas, Pacheco y Villalobos, 2004).

\section{Ejercicio del liderazgo}

Es la capacidad de ejercer influencia sobre los miembros del centro educativo, así como lograr la consecución de los objetivos organizacionales (Vargas, 2010; Coronel, 2005). 
Análisis de la formación profesional de los directores graduados y las directoras graduadas de la carrera de Administración Educativa del CIDE-UNA

lleana Vargas Jiménez

\section{Los procesos de comunicación}

Son los procedimientos que utiliza el director o la directora escolar para comunicarse con los miembros de la comunidad escolar (Latorre, 2006; Jabif, 2004).

\section{La toma de decisiones}

Es el proceso de ejercer autoridad formal que le concede el puesto. Para ello resuelve tomando decisiones de carácter administrativo (Villarreal, 2005).

Para operacionalizar las variables del estudio se elaboró una matriz, la cual funge como la guía sobre la que se trabajó en la aplicación del cuestionario y se transformó en indicadores, ítem con selección única, afirmaciones de acuerdo, grados de importancia y satisfacción e interpretación para el indicador. El cuestionario se operacionaliza en información sociodemográfica, aspectos del plan de estudios, liderazgo, toma de decisiones, comunicación, formación académica para la adaptación de cambios, habilidades y destrezas, valores y actitudes y formación profesional recibida en la práctica supervisada.

\section{Descripción de las técnicas de recolección de datos y los instrumentos}

La técnica de recolección de datos es la estrategia que permite establecer contacto con las personas participantes para obtener la información necesaria en función de los objetivos propuestos en la investigación, en congruencia con la metodología y el marco teórico. De tal manera, se pretende trabajar con la técnica de la encuesta y con el instrumento del cuestionario.

Asimismo, cabe mencionar que, acorde con la investigación cuantitativa y la recolección de los datos empíricos, se pretende utilizar el Cuestionario dirigido a directores (el instrumento ha sido solicitado al SINAES [Sistema Nacional de Acreditación de la Educación Superior]). 
Análisis de la formación profesional de los directores graduados y las directoras graduadas de la carrera de Administración Educativa del CIDE-UNA

lleana Vargas Jiménez

El cuestionario ha sido adaptado con las preguntas que importa abarcar desde las variables y dimensiones del estudio, el marco teórico y los objetivos de la investigación.

Para la validación del instrumento este se sometió a un proceso de valoración a partir de las siguientes estrategias:

\section{Juicio de personas expertas}

Es fundamental para obtener evidencia de validez de contenido conforme a los siguientes pasos: revisar cómo se han medido las variables en investigaciones anteriores y elaborar un universo de ítems tan amplio como sea posible para medir las variables en todas sus dimensiones.

\section{Aplicación de una prueba piloto}

Se debe aplicar a un grupo equivalente de la muestra del estudio, con la finalidad de obtener evidencia de validez y confiabilidad y comprobar la adecuación de las instrucciones y si los ítems funcionan de manera adecuada. Es necesario recordar que los resultados obtenidos en esta prueba deben cumplir con algunas características psicométricas como confiabilidad, validez y objetividad.

\section{Descripción de las personas participantes}

El estudio se llevó a cabo en la provincia de Heredia, específicamente en el Centro de Investigación y Docencia en Educación de la Universidad Nacional. Tienen gran relevancia en esta investigación los directores y las directoras que se graduaron de la Licenciatura de Administración Educativa en el periodo 2000-2011.

Para la negociación de entrada se contactó, en primer lugar, a las personas graduadas mediante llamada telefónica para informarles sobre el estudio, saber si deseaban participar, si contaban con correo electrónico, si trabajaban actualmente en un puesto de dirección o si habían laborado en algún momento 
Análisis de la formación profesional de los directores graduados y las directoras graduadas de la carrera de Administración Educativa del CIDE-UNA

lleana Vargas Jiménez

en dicho puesto. Se procedió a enviar el instrumento vía correo electrónico. Finalmente se trabajó con 36 participantes.

\section{Estrategia utilizada para el análisis del dato cuantitativo}

El análisis de los datos y la presentación de los resultados de estos análisis son aspectos fundamentales para realizar una investigación, una presentación completa, precisa y sin sesgos de los resultados en el tratamiento analítico de los datos (Hernández, Fernández y Batista, 2010).

Para poder recolectar la información se envió el cuestionario en línea Lime Survey; este debe ser configurado de acuerdo a los parámetros del sistema, lo que permite que sea agradable para la persona encuestada y seguro en cuanto a la integridad de los datos. Es así que las consultas se dividen en secciones y cada una tiene tipos de preguntas diferentes. Una vez configurada la encuesta en su totalidad se ordenó la base de datos de las personas encuestadas.

Los usuarios, como se les denomina, son las personas que respondieron la encuesta; el sistema les envió una clave especial para que pudieran acceder a la encuesta, solamente la población seleccionada podía visualizar el contenido del instrumento. El sistema envió un recordatorio semanal para los individuos que aún no habían completado la encuesta y las respuestas se almacenaron en una tabla separada, en la cual posteriormente se exportaron los datos como un archivo.xls de Microsoft Excel.

Después de haber recopilado la información de los cuestionarios se procesó estadísticamente con el apoyo del paquete estadístico para ciencias sociales (SPSS) y se procedió al análisis estadístico (Hernández, Fernández y Batista, 2010). Para este proceso se realizaron las siguientes etapas: revisión de los cuestionarios, enumeración de cada uno, codificación de las preguntas del cuestionario y verificación de la coherencia de las respuestas; posteriormente se realizó el diseño de la base de datos en SPSS y se procedió a la digitación de los datos. Seguidamente se llevó a cabo una fase de revisión y verificación para aumentar la confiabilidad del tratamiento de la información, realizando la 
Análisis de la formación profesional de los directores graduados y las directoras graduadas de la carrera de Administración Educativa del CIDE-UNA

limpieza de la base de datos, corrigiendo posibles errores en la digitación de la información y depurando los resultados para presentarlos.

Luego en SPSS se realizaron los análisis descriptivos del caso y de las distribuciones de frecuencias simples y frecuencias acumuladas. Después se ejecutó el análisis de los datos y la presentación de los resultados, aspectos fundamentales para realizar la investigación.

Es así que se presenta el método de consistencia interna Alpha de Cronbach, en el cual se mide la consistencia interna de todos los ítems de manera global e individual calculando el promedio de todos los coeficientes de correlación posibles de las dos mitades. Como se puede observar en el siguiente gráfico, la puntuación indica que en el nivel de importancia y la escala de liderazgo el coeficiente obtenido es de 0,95 , en la escala de satisfacción el coeficiente es de 0,959 , en la escala de toma de decisiones la importancia indica que el coeficiente es de 0,932 y en la escala de satisfacción el coeficiente obtenido es de 0,939 .

En la escala de comunicación el nivel de importancia indica que el coeficiente es de 0,91 y en el nivel de satisfacción el coeficiente es de 0,932. Lo que se demuestra es que el instrumento de medición puede considerarse fiable. En la escala de habilidades y destrezas el nivel de importancia indica que el coeficiente es de 0,834. En la escala de valores y actitudes el nivel de importancia de acuerdo al coeficiente es de 0,807. En la escala de formación profesional el nivel de importancia en cuanto al coeficiente es de 0,927. En la siguiente tabla se indica lo señalado: 
Análisis de la formación profesional de los directores graduados y las directoras graduadas de la carrera de Administración Educativa del CIDE-UNA

Tabla 1. Coeficientes Alfa de Cronbach

\begin{tabular}{|l|c|c|}
\hline Escala & Importancia & Satisfacción \\
\hline Liderazgo & 0,954 & 0,959 \\
\hline Toma de decisiones & 0,932 & 0,939 \\
\hline Comunicación & 0,91 & 0,932 \\
\hline Habilidades y destrezas & 0,834 & \\
\hline Valores y actitudes & 0,807 & \\
\hline Formación profesional & 0,927 & \\
\hline Tabla correlación & & Sig. \\
\hline Correlación de Spearman & 0,761 & $<0.01$ \\
\hline Liderazgo & 0,76 & $<0.01$ \\
\hline Toma de decisiones & 0,698 & $<0.01$ \\
\hline Comunicación & & \\
\hline
\end{tabular}

\begin{tabular}{|c|c|c|c|c|c|}
\hline Escala & Liderazgo & $\begin{array}{c}\text { Toma de } \\
\text { decisiones }\end{array}$ & Comunicación & $\begin{array}{l}\text { Habilidades } \\
\text { y destrezas }\end{array}$ & $\begin{array}{l}\text { Valores y } \\
\text { actitudes }\end{array}$ \\
\hline Liderazgo & 1 & 0,816 & 0,7 & 0,502 & 0,486 \\
\hline Toma de decisiones & 0,816 & 1 & 0,768 & 0,302 & 0,391 \\
\hline Comunicación & 0,7 & 0,768 & 1 & 0,525 & 0,479 \\
\hline Habilidades y destrezas & 0,502 & 0,302 & 0,525 & 1 & 0,745 \\
\hline \multirow[t]{4}{*}{ Valores y actitudes } & 0,486 & 0,391 & 0,479 & 0,745 & 1 \\
\hline & $\operatorname{sig}<0.01$ & & & & \\
\hline & $\operatorname{sig}<0.05$ & & & & \\
\hline & no sig & & & & \\
\hline
\end{tabular}

Fuente: Elaboración propia 
Análisis de la formación profesional de los directores graduados y las directoras graduadas de la carrera de Administración Educativa del CIDE-UNA

lleana Vargas Jiménez

\section{Resultados y análisis}

\section{Sexo, edad y grado académico de las personas entrevistadas}

De las 36 personas que respondieron el cuestionario el $58,3 \%$ (21) son hombres y el restante $41,7 \%$ (15) son mujeres. Las edades se distribuyen de acuerdo al siguiente rango: para los hombres 46 años y para las mujeres 43 años. Asimismo, la mayoría ostenta el grado de licenciatura $(52,8 \%$ y el resto $(47,2 \%)$ el grado de maestría.

\section{Situación laboral}

Al momento de realizar la entrevista el $55,5 \%$ (20) eran directores y directoras, el $22,2 \%$ (8) de los cuales fueron directores y ahora son supervisores, un director regional, profesoras universitarias, docentes escolares y $19,4 \%$ (7) eran asistentes administrativos y una persona era subdirectora.

Me interesaba conocer la opinión de las personas participantes sobre la dificultad de conseguir trabajo para un profesional en su especialidad. Al respecto, un 58,3\% (21) afirmó que fue difícil conseguir empleo, un 36,1\% (13) manifestó que ni difícil ni fácil y dos personas indicaron que fue fácil. Lo anterior evidencia que para la mayoría de las personas encuestadas sí es difícil obtener empleo en la especialidad.

También resulta de interés destacar que el 77,8\% manifestó que utilizaba mucho los conocimientos y las habilidades adquiridas durante la carrera, mientras que el 22,2\% (8) opinó que los utilizaba en algo. Es decir, predomina en la población seleccionada el uso de los conocimientos y las habilidades adquiridos durante su formación profesional.

En la pregunta 7 de la información sociodemográfica ¿cuántos años lleva trabajando en ese lugar? las respuestas fueron variadas, ya que cuatro de ellos tenían menos de un año, tres personas contestaron que un año, dos personas tres años, tres personas tres años, una persona cuatro años, dos personas cinco años, dos personas seis años de laborar, tres personas tenían siete años, 
Análisis de la formación profesional de los directores graduados y las directoras graduadas de la carrera de Administración Educativa del CIDE-UNA

lleana Vargas Jiménez

una persona tenía ocho años, dos personas contaban con diez años de laborar y una persona tenía once años, tal como se demuestra en la tabla 2.

\section{Tabla 2. Respuestas de las personas graduadas acerca de los años que tienen de laborar en la institución}

\begin{tabular}{|l|c|c|}
\hline Años de laborar & $\mathbf{N}^{\circ}$ de personas & Porcentaje \\
\hline Menos de 1 año & 4 & 11,1 \\
\hline 1 año & 3 & 8,3 \\
\hline 2 años & 3 & 8,3 \\
\hline 3 años & 3 & 8,3 \\
\hline 4 años & 1 & 2,8 \\
\hline 5 años & 2 & 5,6 \\
\hline 6 años & 2 & 5,6 \\
\hline 7 años & 3 & 8,3 \\
\hline 8 años & 1 & 2,8 \\
\hline 9 años o más & 14 & 38,8 \\
\hline Total & 36 & 100,0 \\
\hline
\end{tabular}

Fuente: Elaboración propia

\section{Aspectos del plan de estudios según las personas graduadas}

En cuanto a las preguntas sobre el plan de estudios, estas abarcan de la pregunta 10 a la pregunta 13 y la escala empleada es: 5 muy de acuerdo, 4 de acuerdo, 3 indecisa, 2 en desacuerdo y 1 muy en desacuerdo.

Para la pregunta 10, vinculada —como se mencionó anteriormente- a aspectos del plan de estudios (especialmente en la secuencia de los cursos y si estos eran coherentes), en la tabla 3 se presentan las opiniones de las personas graduadas: en desacuerdo cuatro personas, indecisas seis, de acuerdo diecisiete y muy de acuerdo nueve. Los resultados sugieren la existencia de posibles reclamos en relación con la secuencia de los cursos, así como en su coherencia. 
Análisis de la formación profesional de los directores graduados y las directoras graduadas de la carrera de Administración Educativa del CIDE-UNA

lleana Vargas Jiménez

Por otra parte, en la pregunta 11 del cuestionario se le consulta a la persona entrevistada si la formación académica de la carrera la preparó para adaptarse a los cambios dentro de la disciplina en la que se formó. Se presentan en la tabla 3 las opiniones de las personas graduadas: muy en desacuerdo (ninguna persona), en desacuerdo (tres personas), indecisas (tres personas), de acuerdo (catorce personas) y muy de acuerdo (dieciséis personas).

Según los resultados, se puede inferir que la carrera prepara para adaptarse a los cambios dentro de la disciplina profesional, ya que ciertas personas estuvieron en desacuerdo o indecisas, tal como se señaló en los datos anteriores.

En la pregunta 12 del cuestionario, concerniente a si la formación académica recibida dentro del plan de estudios le permite a la persona entrevistada adaptarse a cambios dentro del contexto laboral, se presentan las opiniones en la siguiente tabla: muy en desacuerdo una persona, en desacuerdo dos personas, indecisas dos personas, de acuerdo quince y muy de acuerdo dieciséis, lo que evidencia que una mayoría está entre muy de acuerdo y de acuerdo.

En la pregunta 13, acerca de si el perfil profesional-académico de salida es congruente con el perfil esperado para ejercer profesionalmente, se presentan los resultados de las opiniones: muy en desacuerdo una persona, en desacuerdo dos personas, indecisas siete personas, de acuerdo catorce personas y muy de acuerdo doce personas.

Se puede observar que, según las opiniones de las personas graduadas, las preguntas que se refieren a lo externo de la carrera obtuvieron mayor crítica; no obstante, las preguntas que se refieren a la persona graduada desde su propia formación profesional recibieron una mejor puntuación. 
Análisis de la formación profesional de los directores graduados y las directoras graduadas de la carrera de Administración Educativa del CIDE-UNA

lleana Vargas Jiménez

A continuación se presentan los resultados en la tabla 3 según las preguntas $10,11,12$ y 13.

\section{Tabla 3. Aspectos del plan de estudios según las personas graduadas}

\begin{tabular}{|c|c|c|c|}
\hline Pregunta & Categoría & Frecuencia & Porcentaje \\
\hline \multirow{5}{*}{$\begin{array}{l}\text { 10. ¿La secuencia de los cursos fueron } \\
\text { coherentes? }\end{array}$} & Muy en desacuerdo & 0 & 0 \\
\hline & En desacuerdo & 4 & 11,1 \\
\hline & Indecisa & 6 & 16,7 \\
\hline & De acuerdo & 17 & 47,2 \\
\hline & Muy de acuerdo & 9 & 25,0 \\
\hline \multirow{5}{*}{$\begin{array}{l}\text { 11. ¿La formación académica en la carrera } \\
\text { prepara a la persona para adaptarse a } \\
\text { los cambios dentro de la disciplina en la } \\
\text { que se formó? }\end{array}$} & Muy en desacuerdo & 0 & 0 \\
\hline & En desacuerdo & 3 & 8,3 \\
\hline & Indecisa & 3 & 8,3 \\
\hline & De acuerdo & 14 & 38,9 \\
\hline & Muy de acuerdo & 16 & 44,4 \\
\hline \multirow{5}{*}{$\begin{array}{l}\text { 12. ¿La formación académica recibida } \\
\text { dentro del plan de estudios le permite } \\
\text { adaptarse a cambios dentro del } \\
\text { contexto laboral? }\end{array}$} & Muy en desacuerdo & 1 & 2,8 \\
\hline & En desacuerdo & 2 & 5,6 \\
\hline & Indecisa & 2 & 5,6 \\
\hline & De acuerdo & 15 & 41,7 \\
\hline & Muy de acuerdo & 16 & 44,4 \\
\hline \multirow{5}{*}{$\begin{array}{l}\text { 13. ¿La formación académica recibida } \\
\text { dentro del plan de estudios le permite } \\
\text { adaptarse a cambios dentro del } \\
\text { contexto laboral? }\end{array}$} & Muy en desacuerdo & 1 & 2,8 \\
\hline & En desacuerdo & 2 & 5,6 \\
\hline & Indecisa & 7 & 19,4 \\
\hline & De acuerdo & 14 & 38,9 \\
\hline & Muy de acuerdo & 12 & 33,3 \\
\hline
\end{tabular}

Fuente: Elaboración propia

\section{Resultados para la variable de liderazgo según las personas graduadas}

Se comparan los resultados para la variable de liderazgo con la satisfacción e importancia percibidas por la población estudiantil. Es importante destacar que los resultados demuestran la existencia de diferencias significativas para ambas escalas; por lo tanto la satisfacción y la importancia fueron valoradas 
Análisis de la formación profesional de los directores graduados y las directoras graduadas de la carrera de Administración Educativa del CIDE-UNA

con puntuaciones diferentes, por lo tanto, son las variables que pretendo analizar en esta investigación.

A continuación se señalan los ítems que las personas entrevistadas contestaron de forma diferente en cuanto a satisfacción e importancia:

1. La temática de promoción de desarrollo de sus colaboradores

2. El accionar hacia la realización de esfuerzos tendientes al logro de objetivos institucionales

3. Se concibe la visión institucional como prioridad en la institución.

4. Se concibe la misión institucional como prioridad en la institución.

7. La ética profesional en la formación profesional

10. Involucrar a los padres y las madres de familia en la rendición de cuentas

11. Los estudiantes son parte importante del quehacer institucional.

No obstante, para las restantes preguntas no se exhiben diferencias relevantes en las escalas de importancia y de satisfacción, estas son:

5. El director y la directora como partícipes del proceso curricular de la institución

6. El liderazgo como punto medular de la institución

8. Las personas docentes del centro educativo son prioridad en la agenda del trabajo institucional.

9. La motivación como parte importante del quehacer cotidiano

De acuerdo con la operacionalización de la variable la práctica directiva está enfocada en el liderazgo, que es la capacidad de ejercer influencia sobre los miembros del centro educativo, así como lograr la consecución de los objetivos organizacionales. Para ello se presenta la tabla 4 con sus respectivas preguntas y el puntaje obtenido para las escalas de importante y satisfactorio. 


\section{Tabla 4. Comparación entre la importancia y la satisfacción percibida en cuanto al liderazgo en el plan de estudios. $\mathrm{N}=36$}

\begin{tabular}{|c|c|c|c|c|}
\hline \multicolumn{5}{|c|}{ Liderazgo } \\
\hline \multirow[b]{2}{*}{ Pregunta } & \multicolumn{3}{|c|}{ Puntaje } & \multirow[b]{2}{*}{ Valor $p$} \\
\hline & Importancia & Satisfacción & Diferencia & \\
\hline $\begin{array}{l}\text { 1. La temática de promoción de desarrollo de } \\
\text { sus colaboradores }\end{array}$ & 84,44 & 77,78 & 6,67 & 0,0377 \\
\hline $\begin{array}{l}\text { 2. El accionar hacia la realización de esfuerzos } \\
\text { tendientes al logro de objetivos } \\
\text { institucionales }\end{array}$ & 87,22 & 80,00 & 7,22 & 0,0354 \\
\hline $\begin{array}{l}\text { 3. ¿Se concibe la visión institucional como } \\
\text { prioridad en la institución? }\end{array}$ & 88,89 & 80,56 & 8,33 & 0,0016 \\
\hline $\begin{array}{l}\text { 4. ¿Se concibe la misión institucional como } \\
\text { prioridad en la institución? }\end{array}$ & 87,78 & 82,78 & 5,00 & 0,0179 \\
\hline $\begin{array}{l}\text { 5. El director y la directora como partícipes del } \\
\text { proceso curricular de la institución }\end{array}$ & 86,67 & 83,89 & 2,78 & 0,3242 \\
\hline $\begin{array}{l}\text { 6. El liderazgo como punto medular en la } \\
\text { institución }\end{array}$ & 91,11 & 88,89 & 2,22 & 0,3785 \\
\hline $\begin{array}{l}\text { 7. La ética profesional en la formación } \\
\text { profesional }\end{array}$ & 92,22 & 81,67 & 10,56 & 0,0084 \\
\hline $\begin{array}{l}\text { 8. ¿Las personas docentes del centro } \\
\text { educativo son prioridad en la agenda de } \\
\text { trabajo institucional? }\end{array}$ & 86,11 & 83,89 & 2,22 & 0,4219 \\
\hline $\begin{array}{l}\text { 9. La motivación como parte importante del } \\
\text { quehacer cotidiano }\end{array}$ & 88,89 & 86,11 & 2,78 & 0,2570 \\
\hline $\begin{array}{l}\text { 10. Involucrar a los padres y las madres de } \\
\text { familia en la rendición de cuentas }\end{array}$ & 88,89 & 80,00 & 8,89 & 0,0015 \\
\hline $\begin{array}{l}\text { 11. ¿La población estudiantil es parte } \\
\text { importante del quehacer institucional? }\end{array}$ & 92,78 & 84,44 & 8,33 & 0,0092 \\
\hline
\end{tabular}

Fuente: elaboración propia

Nota: Las cifras en rojo denotan significancia estadística

Para el análisis de los resultados de esta variable concierne valorar las preguntas que son puntualizadas por las personas graduadas como significativas (las que se señalaron con valores rojos en la tabla).

Es importante señalar que el ejercicio del liderazgo se construye colectivamente. Ahora bien, si se observa la pregunta 1 -vinculada con la temática de promoción de desarrollo de las personas colaboradoras-, sobresale la participación de los miembros de la comunidad educativa, un aspecto que es fundamental llevar a cabo. Pareciera que, desde la carrera y según el criterio de las personas egresadas, este aspecto obtuvo una 
Análisis de la formación profesional de los directores graduados y las directoras graduadas de la carrera de Administración Educativa del CIDE-UNA

lleana Vargas Jiménez

valoración favorable, de la misma manera que la pregunta 2, relacionada con la realización de esfuerzos tendientes al logro de objetivos colectivamente; en esta última pregunta obtuvo mejor puntuación la importancia que la satisfacción.

En la pregunta 3 se concibe la visión del centro escolar como prioridad institucional y en la cuatro se concibe su misión como pilar fundamental de la institución. Lo anterior concuerda con lo expuesto por Garbanzo y Orozco (2010) y Vargas, Pacheco y Villalobos (2004), ya que señalaron que la visión, la misión y los objetivos institucionales se construyen con el colectivo institucional.

En la pregunta 7, las personas encuestadas opinaron que la ética profesional es muy importante; no obstante, en el nivel de satisfacción esta fue calificada con una nota más baja (ver tabla 4). Igualmente, para la población del estudio es evidente que involucrar a los padres y las madres de familia para rendir cuentas (pregunta 10) y que los estudiantes sean parte del quehacer institucional (pregunta 11) son fundamentales desde la formación recibida (de la misma manera, la importancia obtuvo mejor puntuación que la satisfacción).

\section{Resultados para la variable de toma de decisiones percibida por las personas graduadas}

La variable de toma de decisiones está comprendida por ocho preguntas. Los resultados se presentan en la tabla 5 donde se manifiestan diferencias relevantes. En los siguientes ítems los resultados exhiben que la satisfacción obtuvo una menor valoración que la importancia:

1. La toma de decisiones está basada en el respeto.

2. La toma de decisiones está basada en el diálogo.

4. Conocimiento de la política educativa nacional

5. De la realidad educativa como prioridad en una institución

7. La toma de decisiones es un tema importante en la formación profesional. 
Análisis de la formación profesional de los directores graduados y las directoras graduadas de la carrera de Administración Educativa del CIDE-UNA

lleana Vargas Jiménez

Por el contrario, en las preguntas 3, 6 y 8, respectivamente, la satisfacción y la importancia tienen la misma relevancia, según las personas encuestadas.

3. Resolver problemas en el ámbito institucional debe ser una prioridad en la institución.

6. Las teorías abordadas en cursos tomaron en cuenta el aspecto de la toma de decisiones administrativas.

8. Las lecturas sobre toma de decisiones permiten mejorar las relaciones humanas institucionales.

Al retomar la operacionalización de la variable la práctica directiva, desde la toma de decisiones, es el proceso de ejercer autoridad formal que le concede el puesto a la persona que dirige, para ello resuelve tomando decisiones de carácter administrativo. Es así que se indican algunas preocupaciones que revelaron las personas graduadas, lo cual representa una oportunidad de mejora dentro del plan de estudios. Por ejemplo, la pregunta 1: ¿La toma de decisiones está basada en el respeto? y la pregunta 2: ¿La toma de decisiones está basada en el diálogo? evidencian que la importancia obtuvo mejor puntuación que la satisfacción, con base en lo cual se infiere que el plan de estudios debería mejorarse.

Asimismo, en la pregunta 4, relacionada con el conocimiento de la política educativa nacional, se evidencia una mejor puntuación para la importancia que para la satisfacción.

En la pregunta 5, que alude al conocimiento de la realidad educativa nacional, sale mejor puntuada la importancia que la satisfacción, se infiere que representa una oportunidad de mejorar desde el plan de estudios.

La pregunta 7 revela que para las personas egresadas la toma de decisiones debe ser un tema importante en la formación profesional. En esta pregunta recibió una mejor puntuación la importancia que la satisfacción (ver tabla 5). 


\section{Tabla 5. Comparación entre la importancia y la satisfacción percibida en cuanto a la toma de decisiones en el plan de estudios. $\mathbf{N}=\mathbf{3 6}$}

\begin{tabular}{|c|c|c|c|c|}
\hline \multirow[b]{2}{*}{ Pregunta } & \multicolumn{2}{|c|}{ Puntaje } & \multirow[b]{2}{*}{ Diferencia } & \multirow[b]{2}{*}{ Valor $p$} \\
\hline & Importancia & Satisfacción & & \\
\hline $\begin{array}{l}\text { 1. ¿La toma de decisiones está basada en el } \\
\text { respeto? }\end{array}$ & 88,89 & 83,33 & 5,56 & 0,0394 \\
\hline $\begin{array}{l}\text { 2. ¿La toma de decisiones está basada en el } \\
\text { diálogo? }\end{array}$ & 90,56 & 84,44 & 6,11 & 0,0142 \\
\hline $\begin{array}{l}\text { 3. ¿Resolver problemas en el ámbito institucional } \\
\text { debe ser una prioridad en la institución? }\end{array}$ & 88,89 & 85,56 & 3,33 & 0,1834 \\
\hline 4. Conocimiento de la política educativa nacional & 89,44 & 80,56 & 8,89 & 0,0032 \\
\hline $\begin{array}{l}\text { 5. Conocimiento de la realidad educativa como } \\
\text { prioridad en una institución }\end{array}$ & 90,56 & 85,56 & 5,00 & 0,0480 \\
\hline $\begin{array}{l}\text { 6. ¿Las teorías abordadas en cursos tomaban en } \\
\text { cuenta el aspecto de la toma de decisiones? }\end{array}$ & 84,44 & 81,67 & 2,78 & 0,2009 \\
\hline $\begin{array}{l}\text { 7. ¿La toma de decisiones es un tema importante } \\
\text { en la formación profesional? }\end{array}$ & 92,78 & 83,33 & 9,44 & 0,0037 \\
\hline $\begin{array}{l}\text { 8. ¿Las lecturas sobre toma de decisiones } \\
\text { permiten mejorar las relaciones humanas } \\
\text { institucionales? }\end{array}$ & 81,11 & 79,44 & 1,67 & 0,5710 \\
\hline
\end{tabular}

Fuente: elaboración propia

Nota: Las cifras en rojo denotan significancia estadística

Según el análisis de los resultados para la variable de toma de decisiones la escala de importancia obtuvo una mejor valoración que la escala de satisfacción. A pesar de que la satisfacción fue valorada en escala de 80 , la pregunta final acerca de si las lecturas sobre la toma de decisiones permiten mejorar las relaciones humanas institucionales se puntúa como la más baja, especialmente en lo concerniente a la satisfacción; parece que esto debe ser mejorado sustancialmente desde el plan de estudios de la carrera.

\section{Resultados para la variable de comunicación percibida por las personas graduadas}

La variable de comunicación está comprendida por nueve preguntas. Es la variable que presenta menos diferencias entre el nivel de importancia y el de satisfacción. Las preguntas 1,7 y 9 son las que se señalan como diferentes, mientras que las restantes preguntas, $2,3,4,5,6$ y 8 , no representan una diferencia relevante. 
Análisis de la formación profesional de los directores graduados y las directoras graduadas de la carrera de Administración Educativa del CIDE-UNA

1. La comunicación asertiva en la resolución de conflictos

7. La población estudiantil, las y los docentes y las madres y los padres de familia son los agentes clave para la buena comunicación.

9. Busca actualización continua.

Las restantes preguntas: 2, 3, 4, 5, 6 y 8 no representan una diferencia relevante, por lo que cabe señalar que no existe desacuerdo entre las personas entrevistadas sobre la importancia y la satisfacción.

2. La teoría estudiada en cursos permite comprender el proceso de comunicación.

3. Las lecturas recomendadas acerca del proceso administrativo son adecuadas para el contexto actual.

4. Los cursos fueron abordados con respeto y comunicación asertiva, según la misión de la unidad académica.

5. El ambiente y la relación entre profesor y estudiante universitario fue respetuosa y cordial, según la política institucional.

6. Las relaciones humanas institucionales son prioridad en la agenda institucional.

8. La cotidianidad institucional permite una adecuada comunicación institucional.

Al retomar la operacionalización de la variable, la práctica directiva, desde los procesos de comunicación, es el procedimiento que utilizan el director o la directora escolares para comunicarse con los miembros de la comunidad escolar. Tal como se observa en la tabla 16 los datos contrastan al comparar la importancia y la satisfacción percibidas por la población estudiantil.

La comparación entre la importancia y la satisfacción dentro del plan de estudios evidencia que esta no demostró diferencias significativas, tal como se presenta en la tabla 6. 


\section{Tabla 6. Comparación entre la importancia y la satisfacción percibida en cuanto a la comunicación en el plan de estudios. $\mathbf{N}=36$}

\begin{tabular}{|c|c|c|c|c|}
\hline & \multicolumn{2}{|c|}{ Puntaje } & \multirow[b]{2}{*}{ Diferencia } & \multirow[b]{2}{*}{ Valor $p$} \\
\hline Pregunta & Importancia & Satisfacción & & \\
\hline $\begin{array}{l}\text { 1. ¿La comunicación fue asertiva en la } \\
\text { resolución de problemas? }\end{array}$ & 93,89 & 87,78 & 6,11 & 0,0195 \\
\hline $\begin{array}{l}\text { 2. ¿La teoría estudiada en cursos permite } \\
\text { comprender el proceso de comunicación? }\end{array}$ & 82,78 & 81,11 & 1,67 & 0,5392 \\
\hline $\begin{array}{l}\text { 3. ¿Las lecturas recomendadas acerca del } \\
\text { proceso administrativo son adecuadas } \\
\text { para el contexto actual? }\end{array}$ & 79,44 & 74,44 & 5,00 & 0,0595 \\
\hline $\begin{array}{l}\text { 4. ¿Los cursos fueron abordados con } \\
\text { respeto y comunicación asertiva, según la } \\
\text { misión y la visión de la unidad } \\
\text { académica? }\end{array}$ & 88,89 & 87,78 & 1,11 & 0,6239 \\
\hline $\begin{array}{l}\text { 5. ¿La relación profesor-estudiante } \\
\text { universitario fue respetuosa y cordial, } \\
\text { según la política institucional? }\end{array}$ & 91,11 & 89,44 & 1,67 & 0,3731 \\
\hline $\begin{array}{l}\text { 6. ¿Las relaciones humanas institucionales } \\
\text { son una prioridad en la agenda } \\
\text { institucional? }\end{array}$ & 92,78 & 90,00 & 2,78 & 0,1336 \\
\hline $\begin{array}{l}\text { 7. ¿La población estudiantil, las personas } \\
\text { docentes y las madres y los padres de } \\
\text { familia son los agentes clave para la } \\
\text { buena comunicación? }\end{array}$ & 93,89 & 88,89 & 5,00 & 0,0179 \\
\hline $\begin{array}{l}\text { 8. ¿La cotidianidad institucional permite una } \\
\text { adecuada comunicación institucional? }\end{array}$ & 89,44 & 86,11 & 3,33 & 0,1356 \\
\hline 9. ¿Busca actualización continua? & 90,00 & 82,22 & 7,78 & 0,0063 \\
\hline
\end{tabular}

Fuente: elaboración propia

Nota: Las cifras en rojo denotan significancia estadística

Según los resultados, esta variable es la que tiene menos diferencias, pues solamente aparecen tres ítems. La primera pregunta, relacionada con la comunicación asertiva en la resolución de problemas, exhibe una diferencia entre el nivel de importancia y el de satisfacción, ya que el primero es mayor que el segundo.

Asimismo, en la pregunta 7: ¿Las personas docentes, la población estudiantil y los padres y las madres de familia son los agentes clave para la buena comunicación?, aparece evaluada como relevante, aunque con un puntaje bajo en cuanto a satisfacción. 
Análisis de la formación profesional de los directores graduados y las directoras graduadas de la carrera de Administración Educativa del CIDE-UNA

lleana Vargas Jiménez

Para las restantes preguntas, al no existir diferencias importantes, se determinó que las personas encuestadas consideraron un mismo puntaje para el nivel de importancia y el de satisfacción.

Además, en la pregunta 9: ¿Busca actualización continua?, el bajo nivel de satisfacción, en comparación con el de importancia, es relevante. Este es un dato significativo para la carrera, ya que debe mantener a sus egresados y egresadas en permanente actualización profesional.

Comparación de importancia para las variables liderazgo, toma de decisiones y comunicación

La figura 1 presenta la comparación entre las dimensiones de liderazgo, toma de decisiones y comunicación desde la opinión de las personas graduadas en cuanto al nivel de importancia. Los resultados evidencian correspondencia con la teoría de la investigación, puesto que el liderazgo es la dimensión que obtuvo mejor puntuación, tal como se ha reiterado en párrafos anteriores. Además, en segundo lugar está la variable de la comunicación y en tercer lugar la variable de toma de decisiones.

Las tres variables salen por arriba de 80, por lo que se deben realizar esfuerzos por mejorar las temáticas dentro del plan de estudios.

Figura 1. Comparación de las dimensiones de liderazgo, toma de decisiones y comunicación en cuanto a la importancia

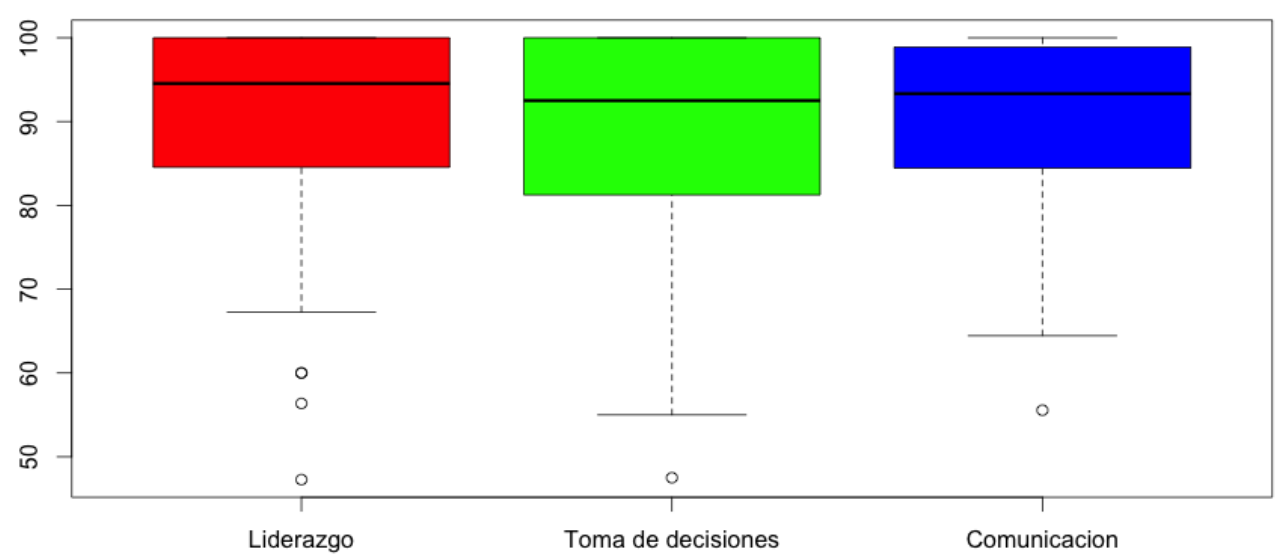

Fuente: elaboración propia 
Análisis de la formación profesional de los directores graduados y las directoras graduadas de la carrera de Administración Educativa del CIDE-UNA

lleana Vargas Jiménez

\section{Comparación de satisfacción para las variables liderazgo, toma de} decisiones y comunicación

La figura 2 muestra la comparación entre las escalas puntuadas en el cuestionario para las variables de liderazgo, toma de decisiones y comunicación. Desde el nivel de satisfacción se puede apreciar que la escala de comunicación es la que obtuvo mejor puntuación, seguida de la de toma de decisiones. También resulta de interés que la población entrevistada haya calificado el liderazgo con la menor puntuación en la escala de satisfacción.

Esto representa una oportunidad de mejora del plan de estudios de la carrera, por lo que se debe fortalecer el área de liderazgo, un aspecto esencial que es necesario desarrollar de manera eficaz en la carrera.

Figura 2. Comparación entre las dimensiones de liderazgo, toma de decisiones y comunicación en cuanto a la satisfacción

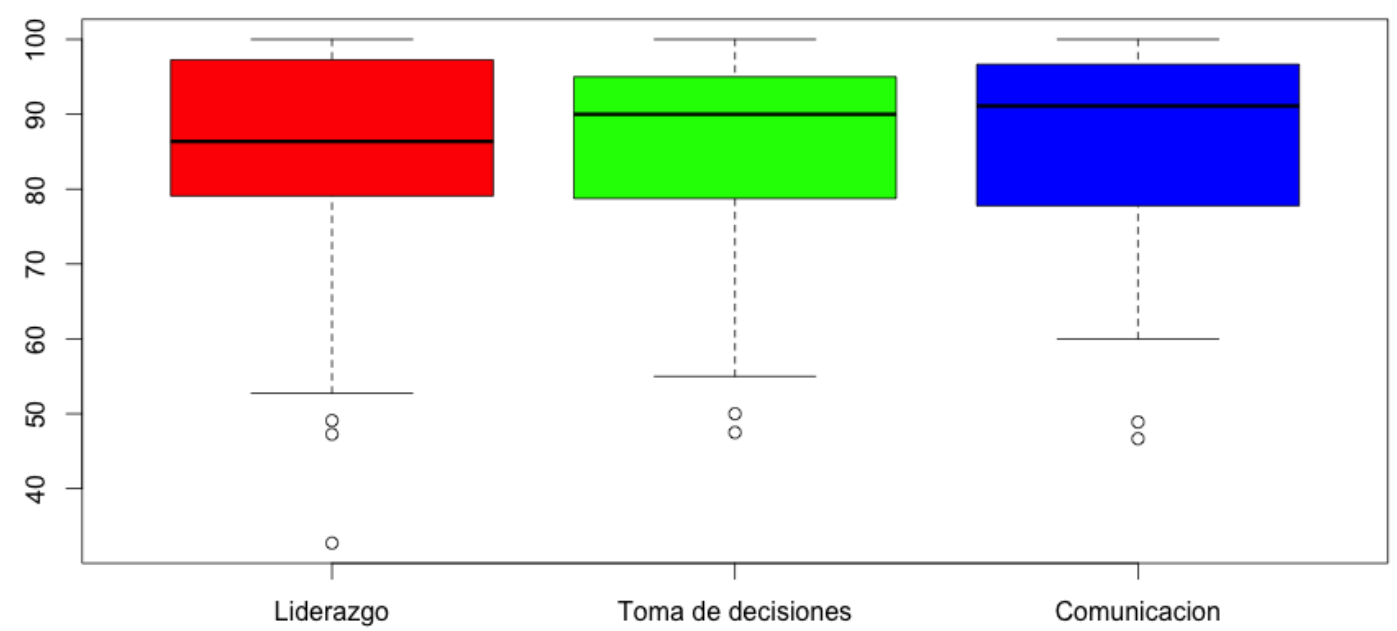

Fuente: elaboración propia

Tal como se ha explicado en párrafos anteriores, y retomando el análisis de los resultados, para las personas graduadas de la carrera resulta muy importante lo relacionado con las variables del estudio: el liderazgo, la toma de decisiones y la comunicación para un buen desempeño profesional. Sin embargo, desde el punto de vista de la satisfacción, las tres variables podrían ser retomadas 
Análisis de la formación profesional de los directores graduados y las directoras graduadas de la carrera de Administración Educativa del CIDE-UNA

lleana Vargas Jiménez

desde el plan de estudios de la carrera, ya que, aunque salen bien puntuadas (más de 80), sería importante replantearlas desde el plan de estudios.

En cuanto a la satisfacción, la variable de liderazgo es la que sale con menor puntuación, por lo que cabe inferir que se deben realizar esfuerzos desde la carrera para mejorarla sustancialmente, seguida de la toma de decisiones. En contraposición, la variable de comunicación es la que obtuvo una mejor valoración desde la satisfacción.

A continuación se presentan los principales hallazgos encontrados en los resultados analizados.

\section{Análisis y discusión de los principales hallazgos}

Según los resultados encontrados y el análisis efectuado en esta fase se presentan los principales hallazgos:

- Las personas graduadas manifestaron que han logrado acumular experiencia en el ejercicio de la administración educativa; asimismo, consideraron que fue difícil conseguir empleo en su especialidad, lo que podría indicar que cada vez se hace más difícil obtener un puesto de dirección. Se podría inferir que esto se debe al bono demográfico señalado en el informe Estado de la Educación 2, en el que se manifiesta que

Las tendencias demográficas reducirán la población en edad escolar, la disminución en la cantidad de niños, niñas y jóvenes en edad escolar incide en la matrícula. Lo que significa es que la transición demográfica es el resultado de una disminución sustancial de la tasa de natalidad (2008, p. 49).

- Además, los resultados reflejan que la mayoría de las personas graduadas manifestó que sí utilizaba las habilidades y los conocimientos adquiridos durante su formación profesional en la carrera; sin embargo, existe un porcentaje de personas entrevistadas que opinó que utilizaba las habilidades y 
Análisis de la formación profesional de los directores graduados y las directoras graduadas de la carrera de Administración Educativa del CIDE-UNA

lleana Vargas Jiménez

los conocimientos adquiridos en "algo", esto quiere decir que existe cierto vacío en el plan de estudios que las autoridades académicas correspondientes deberían tomar en cuenta.

- Las opiniones manifestadas en cuanto a si la carrera preparó a la persona encuestada para adaptarse a cambios dentro de la disciplina se orientan mayoritariamente hacia una valoración positiva («muy de acuerdo» y «de acuerdo»). Igual calificación reciben las opiniones manifestadas en cuanto a si la carrera le permitió a la persona entrevistada adaptarse a los cambios dentro del contexto laboral.

- En cuanto al plan de estudios y a las variables de la investigación -el ejercicio del liderazgo, la toma de decisiones y los procesos de comunicación, en los niveles de satisfacción y de importancia la mayoría de las personas estableció una valoración positiva, pero no cabe duda de que algunos puntos merecen especial consideración.

- En términos generales, las personas graduadas consideraron que el liderazgo en el plan de estudios, en cuanto al nivel de importancia, se ubica en la escala de muy importante. Las preguntas que se presentan a continuación son las que las personas puntuaron con un mayor grado de desacuerdo en los niveles de importancia y de satisfacción:

1. La temática de la promoción del desarrollo profesional de sus colaboradores

2. El accionar hacia la realización de esfuerzos tendientes al logro de objetivos institucionales

3. ¿Se concibe la visión institucional como prioridad en la institución?

4. ¿Se concibe la misión institucional como prioridad en la institución?

7. La ética profesional en la formación profesional

10. Involucrar a los padres y las madres de familia en la rendición de cuentas 
Análisis de la formación profesional de los directores graduados y las directoras graduadas de la carrera de Administración Educativa del CIDE-UNA

11. ¿La población estudiantil es parte importante del quehacer institucional?

Ahora bien, las preguntas que representan un mayor grado de acuerdo entre la satisfacción y la importancia son las siguientes:

1. El director y la directora como partícipes del proceso curricular de la institución

2. El liderazgo como punto medular en la institución

8. ¿El personal docente del centro educativo es una prioridad en la agenda de trabajo institucional?

9. La motivación como parte importante del quehacer cotidiano.

Para la variable de toma de decisiones en el plan de estudios se presentan las preguntas con una mayor diferencia de puntuación entre el nivel de importancia y el de satisfacción (ver tabla 5):

1. ¿La toma de decisiones está basada en el respeto?

2. ¿La toma de decisiones está basada en el diálogo?

4. Conocimiento de la política educativa nacional

5. Conocimiento de la realidad educativa como prioridad en una institución.

7. ¿La toma de decisiones es un tema importante en la formación profesional?

Estas diferencias entre el nivel de importancia y el de satisfacción resultan relevantes para generar mejoras en la carrera.

Sin embargo, las preguntas que no resultaron diferentes en el nivel de importancia y el de satisfacción son:

3. ¿Resolver problemas en el ámbito institucional debe ser una prioridad en la institución?

6. ¿Las teorías abordadas en los cursos tomaron en cuenta el aspecto de toma de decisiones administrativas? 
Análisis de la formación profesional de los directores graduados y las directoras graduadas de la carrera de Administración Educativa del CIDE-UNA

8. ¿Las lecturas sobre toma de decisiones permiten mejorar las relaciones humanas institucionales?

- Para la variable de comunicación resulta interesante mencionar que fue la dimensión con menos diferencias significativas respecto al nivel de satisfacción y al de importancia.

Las preguntas que se presentan a continuación se puntúan como diferentes en los niveles de importancia y de satisfacción:

1. La comunicación asertiva en la resolución de conflictos

7. ¿La población estudiantil, el personal docente y las madres y los padres de familia son agentes clave para la buena comunicación?

9. ¿Busca actualización continua?

Por otra parte, las preguntas que no resultaron relevantes, tanto en el nivel de importancia como en el de satisfacción, son las expuestas a continuación:

2. ¿La teoría estudiada en los cursos permite comprender el proceso de comunicación?

3. ¿Las lecturas recomendadas acerca del proceso administrativo son adecuadas para el contexto actual?

4. ¿Los cursos fueron abordados con respeto y comunicación asertiva según la misión de la unidad académica?

5. ¿La relación entre profesor-estudiante universitario fue respetuosa y cordial según la política institucional?

6. ¿Las relaciones humanas institucionales son una prioridad en la agenda institucional?

8. ¿La cotidianidad institucional permite una adecuada comunicación institucional?

- Según los resultados, en cuanto al plan de estudios de Administración Educativa del CIDE-UNA, la carrera está posicionada en la comunidad nacional, sin embargo, se deben realizar esfuerzos en lo relativo a la práctica 
Análisis de la formación profesional de los directores graduados y las directoras graduadas de la carrera de Administración Educativa del CIDE-UNA

lleana Vargas Jiménez

pedagógica desde el salón de clases. Les corresponde a la población docente y a las autoridades de la unidad académica reflexionar y determinar qué tipo de formación es la que tiene significado para los estudiantes universitarios. Esta podría ser una oportunidad para replantear y mejorar el plan de estudios de la carrera, y para esto la universidad y, por ende, la unidad académica deben brindar una oferta excelente de Administración Educativa que las personas graduadas no solo consideren importante, sino también fundamental a nivel académico, personal y profesional y en las habilidades y actitudes, sin olvidar que el perfil profesional deberá responder de manera articulada a la práctica directiva, en conjunto con la formación profesional recibida y que demanda la sociedad costarricense.

- Existe relación entre la formación profesional recibida y la práctica directiva escolar desde las dimensiones del liderazgo, de la toma de decisiones y de los procesos de comunicación.

- La mayoría de las personas egresadas aceptaron que utilizaban los conocimientos adquiridos a lo largo de la carrera, aunque otras señalaron que los utilizaban en algo.

- Se rechaza la hipótesis de investigación que se mencionó inicialmente, ya que, según los resultados de la indagación, el plan de estudios de la carrera de Administración Educativa CIDE-UNA sí proporciona los requerimientos necesarios para que el director o la directora desempeñe una adecuada práctica educativa y desde la mirada de los actores involucrados.

- Se desprende del análisis que la formación profesional de los directores y las directoras desde la universidad es fundamental y tiene vinculación con la comunidad educativa en la que está inmersa la dirección educativa. Es así como el plan de estudios debe responder a ejes articulados, no como un paquete de cursos aislados, sino por medio de la coherencia en la estructura curricular. Queda demostrado que la carrera cumple con un perfil profesional y 
Análisis de la formación profesional de los directores graduados y las directoras graduadas de la carrera de Administración Educativa del CIDE-UNA

un plan de estudios ofertado con los que las personas egresadas manifiestan sentirse satisfechas, por lo que es necesario que los directores y las directoras los apliquen pertinentemente en sus instituciones.

\section{Conclusiones}

A partir del análisis de los resultados se presentan las principales conclusiones, teniendo en cuenta el problema, las preguntas de investigación y los objetivos.

- Se concluye que los directores y las directoras deben ejercer un buen liderazgo, el cual se relaciona con la toma de decisiones y los procesos de comunicación. En este sentido, la formación profesional recibida fue satisfactoria e importante.

- Los aprendizajes logrados en las personas graduadas a lo largo de la carrera inciden en su desempeño profesional.

- A partir de la participación de la población encuestada, y con base en el criterio manifestado, se puede afirmar que una gran mayoría externó una opinión muy favorable en cuanto a la formación profesional recibida. No obstante, es menor el nivel de satisfacción que el de importancia, y esto repercute en el plan de estudios de la carrera de Administración Educativa del CIDE, lo que podría representar una oportunidad para mejorar en cuanto a la carrera se refiere.

- Se evidencia que el director o la directora escolar es la persona responsable de generar en su institución el mejoramiento educativo, tal como se mencionó de acuerdo con los autores estudiados. Asimismo, se concluye que un buen desempeño administrativo es determinante para el logro institucional.

- La hipótesis de investigación se rechaza, ya que se afirma que el plan de estudios de la carrera de Administración Educativa del CIDE sí proporciona los requerimientos necesarios para que el director o la directora desempeñe una adecuada práctica directiva desde la mirada de los actores involucrados en el estudio. 
Análisis de la formación profesional de los directores graduados y las directoras graduadas de la carrera de Administración Educativa del CIDE-UNA

lleana Vargas Jiménez

\section{Referencias}

Angulo, S., Hidalgo, J. A., Martínez, M. I., Mora, M. y Ramírez, R. G. (2009). Necesidades de los profesionales de la Administración Educativa para responder a las demandas del siglo XXI (seminario de graduación). Facultad de Educación, Universidad de Costa Rica. San José, Costa Rica.

Barrera, F., Fasih, T. \& Patrinos, H. (2009). Decentralized Decision-Making in Schools. The Theory and Evidence on School-Based Management. [La descentralización de la toma de decisiones en la escuela. La teoría y la evidencia en la administración escolar]. Washington, DC.: The World Bank. doi: 10.1596/978-0-8213-7969-1

Cano, J. (2009). Evolución de la administración y la gestión escolar. Tendencias Pedagógicas, 14, 159-168. Recuperado de http://dialnet.unirioja.es/servlet/articulo?codigo $=3002738$

Concha, C. (2007). Claves para la formación de directivos de instituciones escolares. REICE-Revista Electrónica Iberoamericana sobre calidad, eficacia y cambio en Educación, 5(5), 133-138. Recuperado de http://dialnet.unirioja.es/servlet/articulo?codigo $=2515079$

Coronel, J. M. (2005). El liderazgo del profesorado en las organizaciones educativas: temáticas para su análisis e investigación. Revista Española de Pedagogía, 63(232), 471-490.

Cuenca, R. (2005). La formación docente en América Latina y el Caribe, Tensiones, tendencia y propuestas. Recuperado de http://www.dfpd.edu.uy/cfe/docentes/atd/proy_ley_educ/form_doc_americ a_latina_caribe\%5B1\%5D.pdf

Cuenca, R. (2012). Las múltiples identidades profesionales de la docencia. Conferencia presentada en el III Congreso Pedagógico «Formación profesional del educador y la educadora: Una responsabilidad Compartida». San José, Costa Rica.

Cunningham, W. G. \& Sherman, W. H. (2008). Effective Internships: Building Bridges between Theory and Practice. [Práctica efectiva: Construyendo puentes entre la teoría y la práctica]. Educational Forum, 72(4), 308-318. doi: 10.1080/00131720802361936

Garbanzo, G. y Orozco, V. (2010). Liderazgo para una gestión moderna de procesos educativos. Revista Eduación, 34(1), 15-29.

Hernández, R., Fernández, C. y Baptista, P. (2006). Metodología de la investigación. México: Mc Graw-Hill. 
Análisis de la formación profesional de los directores graduados y las directoras graduadas de la carrera de Administración Educativa del CIDE-UNA

lleana Vargas Jiménez

Hernández, R., Fernández, C. y Batista, P. (2010). Metodología de la investigación ( $5{ }^{\text {ta }}$ ed.). México: Mc Graw Hill.

Jabif, L. (2004). Comunicación. Módulos de formación en competencias para la gestión escolar en contextos de pobreza. Instituto Internacional de Planeamiento de la Educación. UNESCO, Buenos Aires.

Latorre, M. (2006). Nuevas miradas, viejos problemas: Las relaciones entre formación inicial y ejercicio profesional docente. Foro Educacional, 10, 4163.

López, N. (2005). La educación en América Latina, entre el cambio social y la inercia institucional. Revista Galega de Economía, 14(1-2), 1-20. Recuperado http://redalyc.uaemex.mx/src/inicio/ArtPdfRed.jsp?iCve=39114212

Ministerio de Educación Pública (MEP). (2010). Nuestras diez líneas estratégicas: 2010-2014. Al desarrollo por la educación. San José, Costa Rica.

Organización de Estados Iberoamericanos para la Educación, la Ciencia y la Cultura (OEI). (2010). Metas Educativas 2021. La educación que queremos para la generación de los Bicentenarios (informe). Madrid, España: Cudipal Gestión Gráfica.

Pogré, P. (2004). Situación de la formación docente inicial y en servicio en Argentina, Chile y Uruguay. UNESCO, Argentina.

Programa Estado de la Nación en Desarrollo Humano Sostenible (2008). Estado de la Educación 2 (2. ${ }^{a}$ ed.). Consejo Nacional de Rectores. San José, Costa Rica.

Tello, C. G. (2008). Gestionar la escuela en Latinoamérica. Gestión educativa, realidad y política. Revista Iberoamericana de Educación, 45(6), 1-10. Recuperado de http://www.rieoei.org/2367.htm

Vargas, I. (noviembre, 2010). Gestión e innovación en contextos de aprendizaje: Reflexiones y desafíos. Ponencia presentada en el IV Congreso Internacional de Administración de la Educación, Universidad de Costa Rica. San José, Costa Rica.

Vargas, I., Pacheco, V. y Villalobos, E. (2004). Plan de rediseño de la carrera de licenciatura en administración educativa. División de Educación para el Trabajo, Centro de Investigación y Docencia en Educación (CIDE), Universidad Nacional. Heredia, Costa Rica.

Vázquez, R. y Angulo, F. (2006). La caja de pandora de la dirección de centros educativos. Aportaciones de una investigación cualitativa. REICE. Revista 
Análisis de la formación profesional de los directores graduados y las directoras graduadas de la carrera de Administración Educativa del CIDE-UNA

lleana Vargas Jiménez

Iberoamericana sobre calidad, eficacia y cambio en educación, 4(4e), 99112.

Villarreal, E. (2005). La efectividad de la gestión escolar depende de la formación del recurso humano como factor, actor, y promotor del cambio dentro de los procesos, dimensiones y políticas educativas. Revista Iberoamericana de Educación, 37(2), 1-4. 